\title{
Waveform Design for Sum Rate Optimization in Time-Reversal Multiuser Downlink Systems
}

\author{
Yu-Han Yang, Beibei Wang and K. J. Ray Liu \\ Department of Electrical and Computer Engineering, \\ University of Maryland, College Park, MD 20742, USA. \\ \{yhyang, bebewang, kjrliu\}@umd.edu
}

\begin{abstract}
Utilizing channel reciprocity, the traditional timereversal technique boosts the signal-to-noise ratio at the receiver with very low transmitter complexity. However, the large delay spread gives rise to severe inter-symbol interference (ISI) when the data rate is high, and the performance is further degraded in the multiuser downlink due to the inter-user interference (IUI). In this work, we study the weighted sum rate optimization problem in the time-reversal multiuser downlink using waveform design. By exploiting the relation between the allocated power and the SINR targets, an iterative algorithm is proposed to optimize the waveform design through a new power allocation algorithm. Simulation results are shown to demonstrate the superior performance of the proposed algorithm in comparison with other traditional methods.
\end{abstract}

\section{INTRODUCTION}

In wideband communication systems, multipath channel with a very large delay spread results in severe inter-symbol interference (ISI) when the data rate is high. In multiuser downlink communication, one transmitter broadcasts different data streams to many receivers at the same time. Since each receiver is only interested in its own data stream, the unintended data streams result in the inter-user interference (IUI).

In the time-reversal communication system [1], the receiver complexity is very low due to the one-tap detection, that is, the receiver detects the received signal using only one sample instead of more complicated receive equalization. With the channel reciprocity, the transmitter can estimate the channel state information (CSI) through the periodical training signals from the receivers. Among the transmit equalizers, the traditional time-reversal technique is a simple matched-filter (MF) with very low complexity and can maximize the signalto-noise ratio (SNR) at the receiver. However, when the data rate is high, the large delay spread gives rise to severe ISI [2], [3]. Therefore, the performance of MF is limited when the transmitted power is high. Several approaches are proposed to deal with the interference. With multiple-input-single-output (MISO) precoding [2], a zero-forcing (ZF) waveform can be adopted to reduce the ISI. However, ZF does not take the noise into account. In [3], Emami et. al. improve the traditional time-reversal waveform with the minimum mean squared error (MMSE) waveform which suppresses both the ISI and noise, but this MMSE waveform is not optimized under different data rates.

Although the ZF and MMSE waveforms can successfully suppress the ISI, they [2], [3] only consider a single-user scenario with a fixed data rate. In multiuser communication, due to the low complexity compared to nonlinear methods, linear precoding is usually adopted to enhance the intended signal and suppress the IUI. However, to the best of our knowledge, previous works in linear precoding for multiuser MIMO broadcast channels assume flat fading and take no consideration into the ISI introduced by multipath. In this paper, we study the sum rate optimization problem in the time-reversal multiuser downlink with waveform design to simultaneously suppress the ISI and IUI.

In the literature, the sum rate optimization methods for MIMO broadcast channels with linear precoding have been proposed in [4]-[9]. Some of these works [4]-[6] directly optimize the sum rate in the downlink, and some works [5], [7]-[9] exploit the uplink-downlink duality [10]-[12] to alternatingly optimize the sum rate. Such an iterative solution based on virtual uplink first appeared in [13], [14].

In this paper, we assume that the transmitter and each receiver are equipped with a single antenna (multiuser SISO downlink). Despite only one antenna, the abundance of multipath can provide numerous degrees of freedom as virtual antennas, and hence the multipath channel is analogous to a MIMO channel except that each user has a selfinterfering component (namely, ISI). With the well-known uplink-downlink duality, the waveform design for the downlink can be obtained using virtual uplink, given any power allocation. However, the power allocation for sum rate is non-convex for either uplink or downlink. We propose the waveform design through a new power allocation algorithm to alleviate the ISI and IUI. The proposed power allocation algorithm exploits the relation between the allocated power and the SINR targets. Although the resulting sum rate is local optimum, simulation results show that such local optimum can still achieve a much better weighted sum rate performance than traditional methods.

This paper is organized as follows. In Section II, the system model and problem formulation are described. In Section III, we propose an iterative algorithm which alternatingly optimizes between calculating the waveform and the power allocation vector. Finally, the numerical simulation in Section IV illustrates the performance compared with traditional methods, and conclusion is drawn in Section V. 


\section{System MOdEL}

Consider a multiuser downlink multipath channel with $K$ users. The transmitter is assumed to have perfect CSI. The receive signal of the $k$ th user at time $m, y_{k}[m]$, can be written as

$$
y_{k}[m]=\sum_{l} h_{k}[m-l] s[l]+n_{k}[m],
$$

where $s[m]$ is the transmit signal and $h_{k}[m]$ denotes the channel impulse response of user $k$. The channel length of $h_{k}[m]$ is denoted by $L_{k}$, i.e., $h_{k}[m]=0$ for $m<0$ and $m \geq L_{k}$. Writing (1) in a matrix form, we have the receive signal vector of the $k$ th user as

$$
\begin{aligned}
\mathbf{y}_{k} & =\mathbf{H}_{k} \mathbf{s}+\mathbf{n}_{k} \\
& =\mathbf{H}_{k}\left(\sum_{j=1}^{K} \mathbf{u}_{j} \sqrt{p_{j}} x_{j}\right)+\mathbf{n}_{k},
\end{aligned}
$$

where $\mathbf{y}_{k}$ is a $(2 L-1) \times 1$ vector with $L=\max _{k} L_{k}, \mathbf{u}_{j}$ is the transmit waveform, $p_{j}$ is the transmit power allocated to user $j, x_{j}$ is the intended signal for user $j$, and $\mathbf{n}_{k}$ is the additive white Gaussian noise (AWGN) with mean zero and variance $\sigma^{2}$. In (2), $\mathbf{H}_{k}$ is a $(2 L-1) \times L$ Toeplitz matrix with each column vector being the shifted version of $h_{k}[m]$.

In the time-reversal communication system, the users detect the received signal with only one tap. The $L$ th element of $\mathbf{y}_{k}$ can be expressed as

$$
y_{k}[L]=\mathbf{H}_{k}^{(1)}\left(\mathbf{u}_{k} \sqrt{p_{k}} x_{k}+\sum_{j=1, j \neq k}^{K} \mathbf{u}_{j} \sqrt{p_{j}} x_{j}\right)+\left[\mathbf{n}_{k}\right]_{L} .
$$

where $\mathbf{H}_{k}^{(1)}$ denotes the $L$ th row of $\mathbf{H}_{k}$ and $\mathbf{H}_{k}^{(0)}$ consists of all other rows. The summation term indicates the inter-user interference. $\left[\mathbf{n}_{k}\right]_{L}$ denotes the $L$ th element in the vector $\mathbf{n}_{k}$.

In (3), we have explicitly considered the inter-user interference and the noise. However, (3) is only for the scenario with one symbol transmitted. When symbols are transmitted consecutively, then ISI must be considered. The amount of ISI depends on the symbol rate. Therefore, we introduce the decimation ratio $D$, which represents the ratio of the symbol duration to the signal sampling duration. Each element in $\mathbf{y}_{k}$ is a signal sample, and the data symbols are transmitted every $D$ signal samples. Clearly, higher $D$ results in less ISI but lower data rate. In other words, one symbol induces ISI to at most $\lfloor 2(L-1) / D\rfloor$ other symbols. Therefore, with decimation ratio $D$, the channel matrix $\mathbf{H}_{k}$ can be decimated by keeping only $\lfloor 2(L-1) / D\rfloor+1$ rows and deleting the other rows for simplicity.

Therefore, the SINR which considers both ISI and IUI for user $k$ is

$$
\mathrm{SINR}_{k}^{\mathrm{DL}}=\frac{\mathbf{u}_{k}^{H} \mathbf{R}_{k}^{(1)} \mathbf{u}_{k} p_{k}}{\mathbf{u}_{k}^{H} \mathbf{R}_{k}^{(0)} \mathbf{u}_{k} p_{k}+\sum_{j=1, j \neq k}^{K} \mathbf{u}_{j}^{H} \mathbf{R}_{k} \mathbf{u}_{j} p_{j}+\sigma^{2}},
$$

where $\mathbf{R}_{k}^{(1)}=\mathbf{H}_{k}^{(1) H} \mathbf{H}_{k}^{(1)}, \mathbf{R}_{k}^{(0)}=\mathbf{H}_{k}^{(0) H} \mathbf{H}_{k}^{(0)}$, and $\mathbf{R}_{j}=$ $\mathbf{H}_{j}^{H} \mathbf{H}_{j}$. The superscript DL denotes the downlink. The first term and the second term in the denominator denotes ISI and IUI, respectively.

In this paper, we jointly design the waveform $\mathbf{U}=$ $\left[\mathbf{u}_{1}, \ldots, \mathbf{u}_{K}\right]$ and power allocation vector $\mathbf{p}=\left[p_{1}, \ldots, p_{K}\right]^{T}$ to maximize the weighted sum rate subject to a total power constraint $P_{\max }$, i.e.,

$$
\begin{gathered}
\mathcal{P}_{\text {Rate }}^{\mathrm{DL}}: \quad \max _{\mathbf{p}, \mathbf{U}} \sum_{k=1}^{K} \alpha_{k} \log \left(1+\mathrm{SINR}_{k}^{\mathrm{DL}}\right) \\
\text { s.t. } \quad \mathbf{1}^{T} \mathbf{p} \leq P_{\max },
\end{gathered}
$$

where $\alpha_{k}$ denotes the rate weighting coefficient for user $k$.

\section{Iterative Algorithm FOR THE Weighted Sum RATE OPTIMIZATION}

In this section, we develop an iterative algorithm for the weighted sum rate optimization in multiuser downlink multipath channels. The algorithm first solves for the waveforms and power allocation in the virtual uplink system, and then transforms the solution into the original downlink problem.

With the uplink-downlink duality [10], [11], the downlink optimal waveform can be found in the virtual uplink, given any power allocation. However, the power allocation problem for sum rate maximization is non-convex. Hence, we propose a new waterfilling power allocation algorithm for the non-convex objective function.

\section{A. Uplink-Downlink Duality}

The virtual uplink problem is constructed as follows.

$$
\begin{gathered}
\mathcal{P}_{\text {Rate }}^{\mathrm{UL}}: \quad \max _{\mathbf{q}, \mathbf{U}} \sum_{k=1}^{K} \alpha_{k} \log \left(1+\mathrm{SINR}_{k}^{\mathrm{UL}}\right) \\
\text { s.t. } \quad \mathbf{1}^{T} \mathbf{q} \leq P_{\max }
\end{gathered}
$$

where $\mathbf{q}=\left[q_{1}, \ldots, q_{K}\right]^{T}$ is the power allocation in the virtual uplink, the downlink transmit waveform $\mathbf{U}$ becomes the uplink receive waveform, and the uplink SINR for user $k$ is

$$
\operatorname{SINR}_{k}^{\mathrm{UL}}=\frac{\mathbf{u}_{k}^{H} \mathbf{R}_{k}^{(1)} \mathbf{u}_{k} q_{k}}{\mathbf{u}_{k}^{H} \mathbf{R}_{k}^{(0)} \mathbf{u}_{k} q_{k}+\sum_{j=1, j \neq k}^{K} \mathbf{u}_{k}^{H} \mathbf{R}_{j} \mathbf{u}_{k} q_{j}+\sigma^{2}},
$$

where $q_{k}$ is the transmit power of user $k$ in the virtual uplink, and the superscript UL denotes the virtual uplink.

By exploiting the fact that the SINR achievable regions are the same [10] for the two dual problems, we develop an iterative algorithm to solve $\mathcal{P}_{\text {Rate }}^{\mathrm{DL}}$ by first solving $\mathcal{P}_{\text {Rate }}^{\mathrm{UL}}$.

It is now well-known [11] that for given SINR targets $\left\{\gamma_{k}\right\}_{k=1}^{K}$, the duality shows that the minimum required total power for the downlink and its virtual uplink is the same. On the other hand, given a sum power constraint $P_{\max }$, the same SINR region is achievable for both the downlink and its virtual uplink. Therefore, we propose an iterative algorithm to first solve $\mathcal{P}_{\text {Rate }}^{\mathrm{UL}}$ and obtain the solution for Problem $\mathcal{P}_{\text {Rate }}^{\mathrm{DL}}$ The algorithm iterates between computing the waveform $\mathbf{U}$ and solving for the uplink power vector $\mathbf{q}$. After the iteration for virtual uplink is completed, the downlink power vector $\mathbf{p}$ is then calculated with the virtual uplink power vector $\mathbf{q}$. 
It is noteworthy that although the uplink-downlink duality and the waveform design of $\mathbf{U}$ given a fixed power allocation vector is not new, the proposed power allocation is the key element to the iterative sum rate optimization algorithm. Without the power allocation, one cannot iteratively update the waveform $\mathbf{U}$ accordingly.

In the following two subsections, we describe the waveform design and the power allocation algorithm in detail.

\section{B. Waveform Design}

The $\operatorname{SINR}_{k}^{\mathrm{UL}}$ in (7) can also be written as

$$
\operatorname{SINR}_{k}^{\mathrm{UL}}=\frac{q_{k} \mathbf{u}_{k}^{H} \mathbf{R}_{k}^{(1)} \mathbf{u}_{k}}{\mathbf{u}_{k}^{H}\left(q_{k} \mathbf{R}_{k}^{(0)}+\sum_{j=1, j \neq k}^{K} q_{j} \mathbf{R}_{j}+\sigma^{2} \mathbf{I}\right) \mathbf{u}_{k}} .
$$

Given a fixed $\mathbf{q}$, we can choose $\mathbf{u}_{k}$ to be the maximum SINR waveform, which turns out to be the MMSE waveform

$$
\mathbf{u}_{k}^{\mathrm{MMSE}}=c_{k}^{\mathrm{MMSE}}\left(\sum_{j=1}^{K} q_{j} \mathbf{R}_{j}+\sigma^{2} \mathbf{I}\right)^{-1} \mathbf{H}_{k}^{(1) H} .
$$

Here, $c_{k}^{\mathrm{MMSE}}$ is a constant such that the norm of $\mathbf{u}_{k}^{\mathrm{MMSE}}$ is normalized to unit.

\section{Power Allocation Algorithm: Iterative SINR Waterfilling}

Given fixed $\mathbf{U}$, the problem $\mathcal{P}_{\text {Rate }}^{\mathrm{UL}}$ becomes solving the power allocation vector $\mathbf{q}$ given a sum power constraint $P_{\max }$. It can be verified that this problem is non-convex so that the global optimal solution is difficult to search. Therefore, our objective of the power allocation algorithm is to efficiently obtain a near-optimal solution.

We propose a novel power allocation algorithm called iterative SINR waterfilling. Firstly, the optimization variables are changed from $\left\{q_{k}\right\}_{k=1}^{K}$ to $\left\{\gamma_{k}\right\}_{k=1}^{K}$ through the following mapping.

$$
\gamma_{k}=\operatorname{SINR}_{k}^{\mathrm{UL}}=\frac{\mathbf{u}_{k}^{H} \mathbf{R}_{k}^{(1)} \mathbf{u}_{k} q_{k}}{\mathbf{u}_{k}^{H} \mathbf{R}_{k}^{(0)} \mathbf{u}_{k} q_{k}+\sum_{j=1, j \neq k}^{K} \mathbf{u}_{k}^{H} \mathbf{R}_{j} \mathbf{u}_{k} q_{j}+\sigma^{2}}
$$

Let $\mathbf{D}$ be a diagonal matrix with $[\mathbf{D}]_{k k}=\gamma_{k} / \mathbf{u}_{k}^{H} \mathbf{R}_{k}^{(1)} \mathbf{u}_{k}$, and

$$
[\boldsymbol{\Phi}]_{k j}=\left\{\begin{array}{ll}
\mathbf{u}_{j}^{H} \mathbf{R}_{k} \mathbf{u}_{j}, & k \neq j \\
\mathbf{u}_{k}^{H} \mathbf{R}_{k}^{(0)} \mathbf{u}_{k}, & k=j
\end{array} .\right.
$$

Rewriting (10), we can represent the power allocation vector $\mathbf{q}$ in terms of $\left\{\gamma_{k}\right\}_{k=1}^{K}$ by

$$
\mathbf{q}=\left(\mathbf{I}-\mathbf{D} \boldsymbol{\Phi}^{T}\right)^{-1} \mathbf{D} \boldsymbol{\sigma} .
$$

Now, instead of allocating power $\left\{q_{k}\right\}_{k=1}^{K}$, we allocate the SINR targets $\left\{\gamma_{k}\right\}_{k=1}^{K}$.

Thus, the weighted sum rate optimization problem in terms of $\left\{\gamma_{k}\right\}_{k=1}^{K}$ becomes

$$
\begin{aligned}
\max _{\gamma_{1}, \ldots, \gamma_{K}} & \sum_{k=1}^{K} \alpha_{k} \log \left(1+\gamma_{k}\right) \\
\text { s.t. } & \mathbf{1}^{T}\left(\mathbf{I}-\mathbf{D} \boldsymbol{\Phi}^{T}\right)^{-1} \mathbf{D} \boldsymbol{\sigma} \leq P_{\max } \\
& \rho\left(\mathbf{D} \boldsymbol{\Phi}^{T}\right)<1
\end{aligned}
$$

where $\rho(\cdot)$ denotes the spectral radius.

According to the Karush-Kuhn-Tucker (KKT) conditions, the optimum $\gamma_{k}$ must satisfy

$$
\begin{aligned}
& \gamma_{k}=\left(\frac{\alpha_{k}}{\lambda t_{k}}-1\right)^{+} \\
& \mathbf{1}^{T}\left(\mathbf{I}-\mathbf{D} \boldsymbol{\Phi}^{T}\right)^{-1} \mathbf{D} \boldsymbol{\sigma}=P_{\max } \\
& \rho\left(\mathbf{D} \boldsymbol{\Phi}^{T}\right)<1
\end{aligned}
$$

where

$t_{k}=\frac{\mathbf{u}_{k}^{H} \mathbf{R}_{k}^{(1)} \mathbf{u}_{k}}{\gamma_{k}^{2}} \mathbf{1}^{T}\left(\mathbf{I}-\mathbf{D} \boldsymbol{\Phi}^{T}\right)^{-1} \mathbf{D} \mathbf{e}_{k} \mathbf{e}_{k}^{T}\left(\mathbf{I}-\mathbf{D} \boldsymbol{\Phi}^{T}\right)^{-1} \mathbf{D} \boldsymbol{\sigma}$

and $\mathbf{e}_{k}$ is the $k$ th column of a $K \times K$ identity matrix.

Next, in order to solve for $\lambda$, we show the monotonicity of $\lambda$ in the left-hand-side expression of (17) and (18).

Lemma 1: Let $\boldsymbol{\Lambda}$ be a square diagonal matrix with positive diagonal elements, and $\mathrm{S}$ be a square matrix with positive elements. Then $\rho(\boldsymbol{\Lambda} \mathbf{S}) \leq \rho(\boldsymbol{\Lambda}) \rho(\mathbf{S})$.

Proposition 1: $\rho\left(\mathbf{D} \Phi^{T}\right)$ is monotonically decreasing with $\lambda . \mathbf{1}^{T}\left(\mathbf{I}-\mathbf{D} \boldsymbol{\Phi}^{T}\right)^{-1} \mathbf{D} \boldsymbol{\sigma}$ is also monotonically decreasing with $\lambda$ if $\rho\left(\mathbf{D} \Phi^{T}\right)<1$.

Proof: Assume $\hat{\lambda}>\lambda$. From (16), we have $\hat{\gamma_{k}} \leq \gamma_{k}$. $\rho\left(\hat{\mathbf{D}} \mathbf{D}^{-1}\right) \leq 1$. With Lemma 1 ,

$$
\begin{aligned}
\rho\left(\hat{\mathbf{D}} \Phi^{T}\right) & =\rho\left(\hat{\mathbf{D}} \mathbf{D}^{-1} \mathbf{D} \boldsymbol{\Phi}^{T}\right) \\
& \leq \rho\left(\hat{\mathbf{D}} \mathbf{D}^{-1}\right) \rho\left(\mathbf{D} \boldsymbol{\Phi}^{T}\right) \\
& \leq \rho\left(\mathbf{D} \Phi^{T}\right) .
\end{aligned}
$$

Thus, $\rho\left(\mathbf{D} \Phi^{T}\right)$ is monotonically decreasing with $\lambda$.

$$
\begin{aligned}
& \text { If } \rho\left(\mathbf{D} \boldsymbol{\Phi}^{T}\right)<1 \text {, then }\left(\mathbf{I}-\mathbf{D} \boldsymbol{\Phi}^{T}\right)^{-1}=\sum_{r=0}^{\infty}\left(\mathbf{D} \boldsymbol{\Phi}^{T}\right)^{r} . \\
& \qquad \begin{aligned}
\mathbf{1}^{T}\left(\mathbf{I}-\mathbf{D} \boldsymbol{\Phi}^{T}\right)^{-1} \mathbf{D} \boldsymbol{\sigma} & =\mathbf{1}^{T} \sum_{r=0}^{\infty}\left(\mathbf{D} \boldsymbol{\Phi}^{T}\right)^{r} \mathbf{D} \boldsymbol{\sigma} \\
& \geq \mathbf{1}^{T} \sum_{r=0}^{\infty}\left(\hat{\mathbf{D}} \boldsymbol{\Phi}^{T}\right)^{r} \hat{\mathbf{D}} \boldsymbol{\sigma} .
\end{aligned}
\end{aligned}
$$

Thus, $\mathbf{1}^{T}\left(\mathbf{I}-\mathbf{D} \boldsymbol{\Phi}^{T}\right)^{-1} \mathbf{D} \boldsymbol{\sigma}$ is also monotonically decreasing with $\lambda$ if $\rho\left(\mathbf{D} \Phi^{T}\right)<1$.

Since the $\gamma_{k}$ in (16), $\rho\left(\mathbf{D} \Phi^{T}\right)$, and $\mathbf{1}^{T}\left(\mathbf{I}-\mathbf{D} \boldsymbol{\Phi}^{T}\right)^{-1} \mathbf{D} \boldsymbol{\sigma}$ are all monotonic with $\lambda$, the bisection search can be applied to efficiently compute the $\lambda$ such that the power constraint is satisfied. In the one dimensional bisection search, the initial upper bound of $\lambda$ can be set as $\max _{k} \alpha_{k} / t_{k}$ since the SINR targets $\left\{\gamma_{k}\right\}_{k=1}^{K}$ are all zero for $\lambda$ higher than this value. The lower bound can be set as a small positive number, which corresponds to very large values of $\left\{\gamma_{k}\right\}_{k=1}^{K}$.

(16) is a waterfilling-like solution with a feasibility constraint (18) and a nonlinear power constraint (17). The $t_{k}$ can be considered as a modification term to the water level due to the effect of the interference [15]. In solving the optimum $\gamma_{k}$, we can first fix $t_{k}$, and then SINR target $\gamma_{k}$ is found by using bisection search for $\lambda$ and substituting $\lambda$ into (16). The new $\gamma_{k}$ is then used to update $t_{k}$ as in (19). The procedure is 
TABLE I

ITERATIVE SINR WATERFILLING

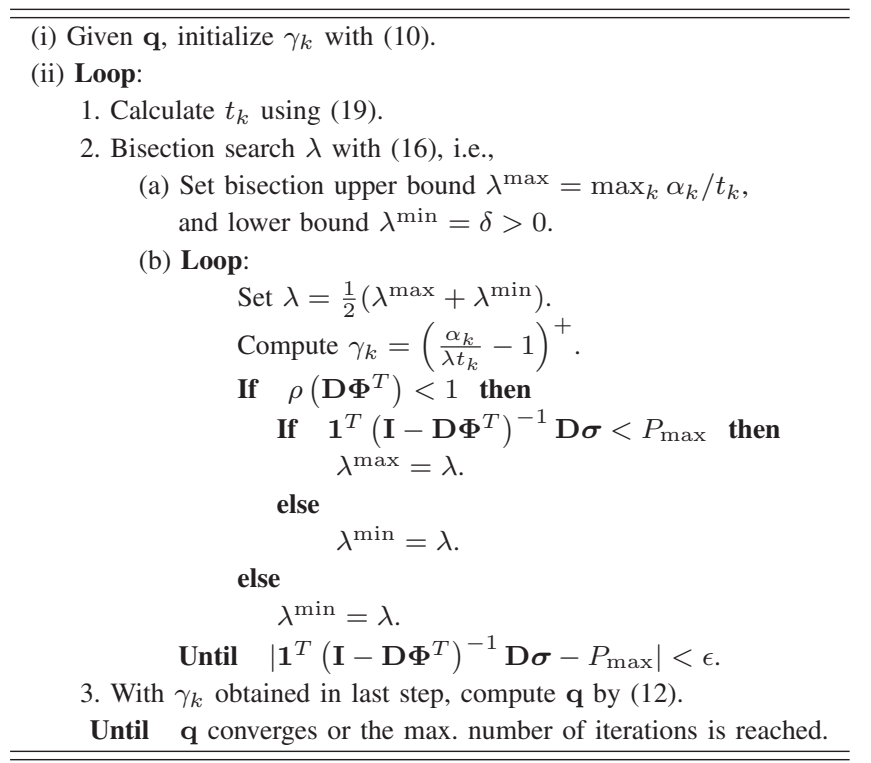

TABLE II

Iterative Weighted Sum Rate Optimization Algorithm

(i) Initialize $q_{k}=P_{\max } / K$.

(ii) Loop (uplink optimization):

1. Calculate $\mathbf{U}$ by (9).

2. Calculate $\mathbf{q}$ using Iterative SINR Waterfilling.

Until $\mathbf{q}$ and $\mathbf{U}$ converges or the max. number of iterations is reached. (iii) Compute $\gamma_{k}$ by (10).

(iv) Obtain downlink power vector $\mathbf{p}$ by (21).

repeated until convergence. The power allocation algorithm is summarized in Table I.

As in [15], we can incorporate a memory term for $\gamma_{k}$ to slow down the update and the convergence can be improved. In the $n$th iteration, the $\gamma_{k}(n)$ can be calculated by

$$
\gamma_{k}(n)=\beta \gamma_{k}^{\text {new }}(n)+(1-\beta) \gamma_{k}(n-1),
$$

where $\gamma_{k}^{\text {new }}(n)$ is the one obtained after the bisection search and $\beta$ is the forgetting factor with $0<\beta<1$.

\section{Iterative Sum Rate Optimization Algorithm}

The iterative sum rate optimization algorithm iterates between calculating the waveform $\mathbf{U}$ using (9) and the power allocation $\mathbf{q}$ using Table I in the virtual uplink. After convergence or maximum number of iterations is reached, we can compute the corresponding achievable SINR targets $\left\{\gamma_{k}\right\}_{k=1}^{K}$ and the downlink power allocation $\mathbf{p}$ can then be obtained similar to (12), i.e.,

$$
\mathbf{p}=(\mathbf{I}-\mathbf{D} \Phi)^{-1} \mathbf{D} \boldsymbol{\sigma}
$$

The proposed iterative algorithm for the weighted sum rate optimization algorithm is summarized in Table II.

\section{Numerical Simulation}

In this section, we use numerical simulation to demonstrate the performance of the proposed iterative sum rate optimization algorithm. In the simulation, each path is assumed to be an i.i.d. complex Gaussian random variable with zero mean and variance of $\frac{1}{2 L}$ per dimension. Figure 1 shows the sum rate performance of a 2 -user system with $L=8, D=2$, $\alpha_{1}=\alpha_{2}=1$. The weighted sum rate performance of a 4-user system is shown in Figure 2, where $L=10, D=4$, and $\alpha_{1}=\alpha_{2}=2, \alpha_{3}=\alpha_{4}=1$. Each rate is averaged over 1000 channel realizations.

In both figures, the forgetting factor $\beta$ is set as $1 / K$. MF denotes the matched-filter; ZF denotes the zero-forcing waveform. The proposed power allocation is the iterative SINR waterfilling algorithm described in Section III-C. The equal power allocation is to split the total power equally to each user, i.e., $p_{k}=P_{\max } / K$. The optimal power allocation is simulated by exhaustive search.

From the figures, it is clear that the proposed power allocation can improve the performance of equal power allocation for all waveform designs, since the iterative SINR waterfilling is able to find a local optimum by taking the channel gains into consideration. The improvement for the MMSE waveform is especially significant at high power region. In Figure 1, the MMSE waveform with the proposed power allocation performs almost the same as the optimal power allocation. We can see that even with the MMSE waveform, which is optimal given any power allocation, the equal power allocation still saturates at high power region.

Note that since the sub-optimal waveforms $\mathrm{MF}$ and $\mathrm{ZF}$ are not related to the power allocation, these methods cannot iteratively optimize between the waveform design and power allocation. For the MMSE with equal power allocation, since the power allocation remains the same, the MMSE waveform cannot update accordingly. Therefore, these methods are not iterative and thus require lower computational complexity compared to the proposed algorithm.

It is well-known [16] that since MF only maximizes the received signal power without considering the interference, it saturates at a lower rate, as shown in both figures. ZF cancels the interference but sacrifices the received signal power resulting in worse performance at low power region. The proposed method strikes a balance between the two by reducing the interference including ISI and IUI, while keeping a high received signal power.

Figure 3 shows typical convergence behaviors of the inner loop (Table I) and outer loop (Table II) of the proposed algorithm. The maximum iteration number of inner loop is set to be 20. Since the power allocation problem is nonconvex, the KKT condition is only necessary, but not sufficient for a global optimum. Thus, the iterative SINR waterfilling only converges to a local optimum, if it converges. However, very fast convergence of the outer loop in is always observed (typically 2 to 8 iterations).

Finally, we note that we have no proof whether our iterative algorithms converge to the global optimum or merely local 


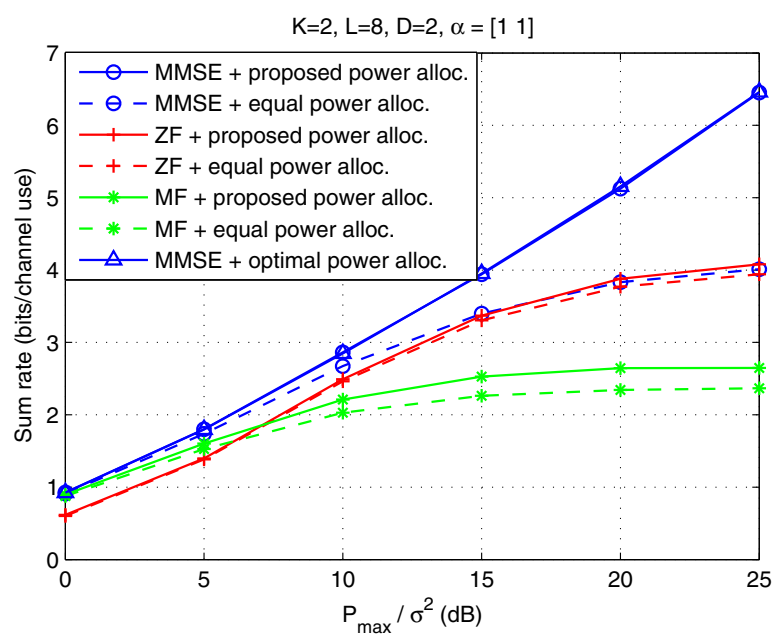

Fig. 1. Sum rate performance for different methods with 2 users.

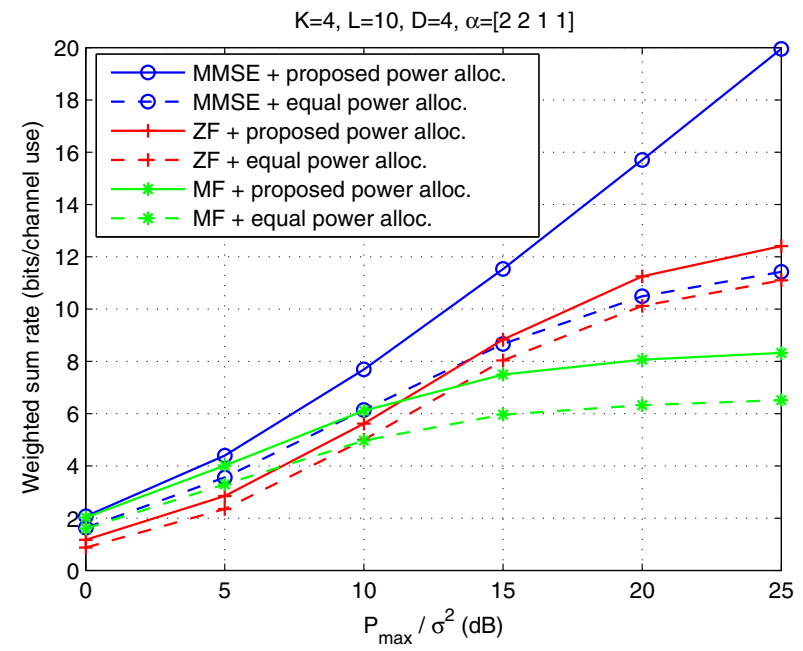

Fig. 2. Weighted sum rate performance for different methods with 4 users. The weighting coefficients are $\alpha_{1}=\alpha_{2}=2$, and $\alpha_{3}=\alpha_{4}=1$.

optima. However, as shown by the simulation results, the local optima still result in much better performance than other traditional methods.

\section{CONCLUSION}

In this paper, we explored the weighted sum rate optimization problem of the waveform design for the time-reversal multiuser downlink communication system. We proposed an iterative algorithm which alternatingly optimizes the waveform and the power allocation. Utilizing the duality for the SINR constrained problems, the proposed iterative SINR waterfilling provides an efficient numerical method for the non-convex power allocation problem.

\section{REFERENCES}

[1] B. Wang, Y. Wu, F. Han, Y.-H. Yang, and K. Liu, "Green wireless communications: A time-reversal paradigm," to appear, IEEE Journal of Selected Areas in Communications, special issue on Energy-Efficient Wireless Communications., 2011.

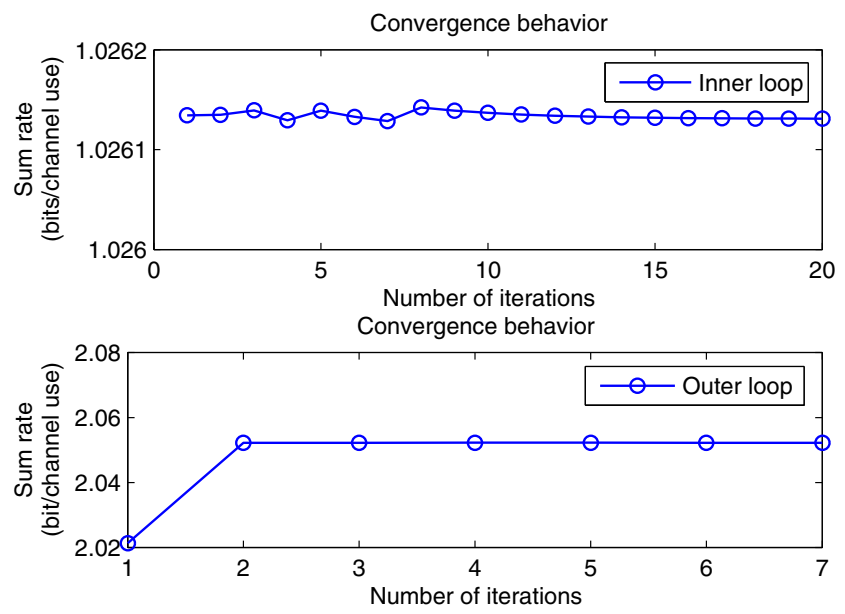

Fig. 3. Convergence behaviors of the inner loop (power allocation) and the outer loop (sum rate optimization).

[2] R. C. Daniels and R. W. Heath, "Improving on time-reversal with MISO precoding," in the Proceedings of the Eighth International Symposium on Wireless Personal Communications Conference, Aalborg, Denmark, 2005.

[3] M. Emami, M. Vu, J. Hansen, A. Paulraj, and G. Papanicolaou, "Matched filtering with rate back-off for low complexity communications in very large delay spread channels," in the 38th Asilomar Conference on Signals, Systems and Computers, 2004, pp. 218-222.

[4] S. S. Christensen, R. Agarwal, E. d. Carvalho, and J. M. Cioffi, "Weighted sum-rate maximization using weighted MMSE for MIMOBC beamforming design," IEEE Trans. Wireless Commun., vol. 7, no. 12, pp. 4792-4799, Dec. 2008.

[5] C. Guthy, W. Utschick, R. Hunger, and M. Joham, "Efficient weighted sum rate maximization with linear precoding," IEEE Trans. Signal Processing, vol. 58, no. 4, pp. 2284-2297, 2010.

[6] M. Stojnic, H. Vikalo, and B. Hassibi, "Rate maximization in multiantenna broadcast channels with linear preprocessing," IEEE Trans. Wireless Commun., vol. 5, no. 9, pp. 2338-2342, Sept. 2006.

[7] S. Shi, M. Schubert, and H. Boche, "Rate optimization for multiuser MIMO systems with linear processing," IEEE Trans. Signal Processing, vol. 56, no. 8, pp. 4020-4030, Aug. 2008.

[8] A. J. Tenenbaum and R. S. Adve, "Improved sum-rate optimization in the multiuser MIMO downlink," in Proc. CISS, Mar. 2008, pp. 984-989.

[9] _ _ "Linear processing and sum throughput in the multiuser MIMO downlink," IEEE Trans. Wireless Commun., vol. 8, no. 5, pp. 2652-2661, May 2009.

[10] D. Tse and P. Viswanath, "Downlink-uplink duality and effective bandwidths," in Proc. IEEE Int. Symp. Inf. Theory (ISIT), Jul. 2002.

[11] M. Schubert and H. Boche, "Solution of the multiuser downlink beamforming problem with individual SINR constraints," IEEE Trans. Veh. Technol., vol. 53, no. 1, pp. 18-28, Jan. 2004.

[12] R. Hunger and M. Joham, "A general rate duality of the mimo multiple access channel and the mimo broadcast channel," in IEEE Global Telecommunications Conference, 2008., Dec 2008, pp. 1 -5.

[13] F. Rashid-Farrokhi, K. J. R. Liu, and L. Tassiulas, "Transmit beamforming and power control for cellular wireless systems," IEEE J. Select. Areas Commun., vol. 16, no. 8, pp. 1437-1450, Oct. 1998.

[14] F. Rashid-Farrokhi, L. Tassiulas, and K. J. R. Liu, "Joint optimal power control and beamforming in wireless networks using antenna arrays," IEEE Trans. Commun., vol. 46, no. 10, pp. 1313-1324, Oct. 1998.

[15] W. Yu, "Multiuser water-filling in the presence of crosstalk," in Information Theory and Applications Workshop, Jan. 2007, pp. 414-420.

[16] F. Dietrich, R. Hunger, M. Joham, and W. Utschick, "Linear precoding over time-varying channels in TDD systems," in Proc. ICASSP'03, vol. 5, 2003, pp. 117-120. 